\title{
Electromagnetic Modeling of High Magnetic Contrast Media using Calderón Preconditioning
}

\author{
Michiel Gossye, Dries Vande Ginste, and Hendrik Rogier \\ Ghent University - imec, Department of Information Technology, IDLab, \\ Electromagnetics Group, Gent, Belgium
}

\begin{abstract}
In this contribution, we present a Calderón preconditioner for a novel singlesource equation to efficiently model electromagnetic scattering problems involving high magnetic contrasts. Through analysis of the spectral properties of the system matrix after discretization, it is shown that this formulation does not break down when high permeabilities are present, which was an unresolved problem of the Calderón preconditioned Poggio-Miller-ChanHarrington-Wu-Tsai method. The adopted discretization scheme, which involves Rao-Wilton-Glisson and Buffa-Christiansen basis functions, allows for an easy integration in existing commercial Method of Moments software. The efficiency and accuracy of the presented method is corroborated by numerical examples.
\end{abstract}

Keywords:

Method of Moments, electromagnetic scattering, Calderón preconditioner, high magnetic contrast

\section{Introduction}

Boundary integral equations (BIEs) play a key role in the numerical modeling of (time-harmonic) electromagnetic (EM) scattering problems. They allow to analyze the interaction of EM fields with piecewise homogeneous media. In general, a faster convergence of the iterative solution is obtained when compared to alternative methods such as the finite element (FE) method, since only the surfaces of the scattering objects are discretized, leading to fewer unknowns. In case the problem includes heterogeneous regions, hybrid FE-BIE formulations may be adopted to take advantage of the efficiency of 
the BIE, and the ability of the FE method to model heterogeneous media [1].

The simulation of homogeneous penetrable bodies is often performed using the Poggio-Miller-Chan-Harrington-Wu-Tsai (PMCHWT) equation [2]. The PMCHWT operator contains the electric field integral operator (EFIO) $\mathcal{T}$, whose spectrum is neither bounded from above nor below. Consequently, the spectrum of the PMCHWT operator inherits these properties, resulting in dense-mesh discretization breakdown [3]. This means that the condition number of the system matrix of the discretized PMCHWT operator will rise as the mesh becomes denser, i.e., as the discretized operator approaches the continuous operator. To resolve these breakdown issues, preconditioners based on the Calderón identities were developed for first-kind BIE problems in [4], and more specifically for the EFIO in [5]. Later, multiplicative Calderón preconditioning has been examined for both the EFIO [6] and the PMCHWT operator [3]. Calderón preconditioners make use of the self-regularizing property of both (continuous) operators, meaning that their squares have eigenvalues that do not converge to zero or infinity. This can be deduced from the Calderón identity $\mathcal{T}^{2}=\mathcal{K}^{2}-\frac{1}{4}$ and the compactness of $\mathcal{K}$, the magnetic field integral operator (MFIO) $[3,6,7]$. Hence, after preconditioning of the EFIO or the PMCHWT operator with itself, a well-conditioned system of equations will be obtained. The transition of the continuous expression to a discrete formulation is possible by introducing the Buffa-Christiansen (BC) basis functions [8], which are defined as a linear combination of Rao-Wilton-Glisson (RWG) functions [9] on the baricentrically refined mesh. As a consequence, both the Calderón preconditioned EFIO (CP-EFIO) and the Calderón preconditioned PMCHWT (CP-PMCHWT) operator are well-conditioned and dense-mesh breakdown is alleviated, at least under the assumption that the dielectric and magnetic contrast between object and exterior region remains low in case of the CP-PMCHWT operator. Indeed, it can be shown [3] that the ratio of the eigenvalue accumulation points of the system matrix of the latter operator equals $\left(k^{e} / k^{i}\right)^{2}$, with $k^{e}$ and $k^{i}$ the wavenumber of the background medium and the scattering object, respectively. This is a measure for the convergence time of the iterative solution [10]. In the numerical study in [11], there is indeed mention of reduced efficiency of the CP-PMCHWT method when large dielectric contrasts are present. Furthermore, convergence problems arise when simulating at resonances and negative material parameters.

In $[12,13,14]$, intrinsically well-conditioned formulations for electromag- 
netic scattering problems were obtained by introducing a local FE approximation of the Dirichlet-to-Neumann (DtN) operator. This DtN operator links the magnetic and electric current on the scatterer surface. Such formulations are known as on-surface radiation condition (OSRC) methods. In the realm of BIEs for 2D transmission problems, a DtN operator was employed in [15], based on the Calderón identities. This DtN operator was approximated by regularizing operators, leading to generalized combined source integral equations (GCSIE). Later, this formalism was extended to 3D electromagnetic transmission problems, resulting in intrinsically well-conditioned integral equations [16].

In [17], a Calderón preconditioned single-source equation, based on the DtN operator, was introduced that does not suffer from dense-mesh breakdown for electromagnetic scattering problems involving arbitrarily high dielectric contrasts (including conductive or negative index media). In this paper, we present a comparable method, based on the Neumann-to-Dirichlet (NtD) operator $\mathcal{Y}$, that does not break down when arbitrarily high permeabilities are present. The NtD operator maps the trace of the magnetic field on the boundary of a scattering object onto the tangential electric field on this boundary. The ill-conditioning of the discretization of $\mathcal{Y}$ will be resolved by making use of the regularizing property of the electric field integral operator $\mathcal{T}$ on the NtD operator [1]. The numerical solution of this novel formulation involves RWG and BC basis functions, which allows for integration in existing numerical solvers that make use of the conventional RWG basis functions.

This paper is structured as follows. In Section 2, the integral representation of the electromagnetic fields is first derived, starting from Maxwell's equations. Next, the derivation of the novel formulation is given. It is compared to the CP-PMCHWT and the method in [17]. In Section 3, the discretization of the proposed method is discussed. Afterwards, the extension to scattering problems with multiple objects is introduced. In Section 4, the eigenvalue spectrum of the corresponding system matrix is derived, to analyze the convergence of the iterative solution. Lastly, in Section 5, numerical examples are given to demonstrate the effectiveness of the proposed method.

\section{Boundary Integral Equation Formulations}

Assume a time-harmonic electromagnetic field $\left(\mathbf{e}^{i}, \mathbf{h}^{i}\right)$ with $e^{j \omega t}$ time dependence. This field impinges on an isotropic and homogeneous dielectric 


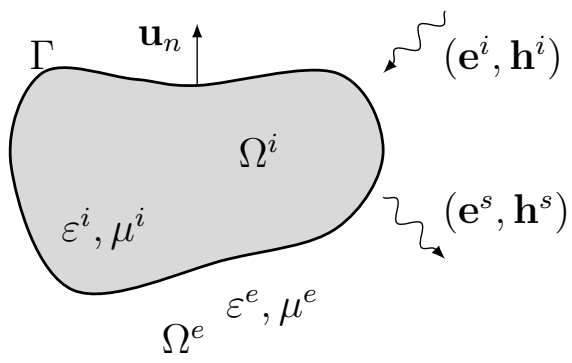

(a) Original problem.

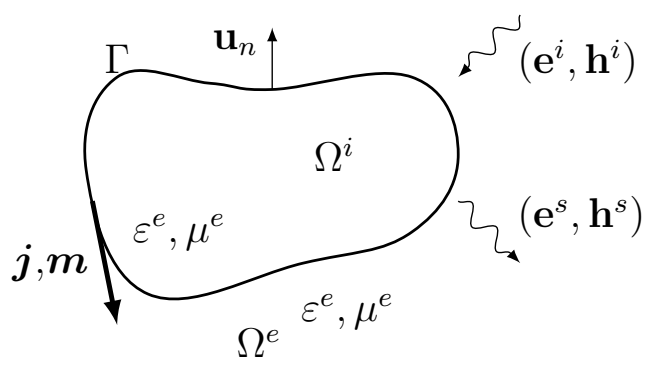

(b) Equivalent problem.

Figure 1: In (a), a homogeneous object $\Omega^{i}$ is embedded in a homogeneous background medium $\Omega^{e}$. An electromagnetic field $\left(\boldsymbol{e}^{i}, \boldsymbol{h}^{i}\right)$ impinges on $\Omega^{i}$, which generates a scattered field $\left(\boldsymbol{e}^{s}, \boldsymbol{h}^{s}\right)$. In (b), we have the equivalent problem, where the object is now filled with the medium of $\Omega^{e}$ and surface currents $\mathbf{j}$ and $\mathbf{m}$ reside on $\Gamma$, generating $\left(\boldsymbol{e}^{s}, \boldsymbol{h}^{s}\right)$.

object $\Omega^{i}$ with permittivity $\epsilon^{i}$ and permeability $\mu^{i}$, embedded in a homogeneous background medium characterized by $\epsilon^{e}$ and $\mu^{e}$. This results in a scattered electromagnetic field $\left(\mathbf{e}^{s}, \mathbf{h}^{s}\right)$ (see Fig. 1(a)). In general, an analytical solution for this scattering problem does not exist. However, a numerical solution can be obtained by first applying the surface equivalence theorem [18], stating that the scattered field in the external region can be generated by equivalent current sources $\mathbf{j}$ and $\mathbf{m}$ on the boundary $\Gamma$ of the object after filling $\Omega^{i}$ with the medium of $\Omega^{e}$ (Fig. 1(b)). In this equivalent problem, which involves current sources $\mathbf{j}$ and $\mathbf{m}$ in a homogeneous and infinite medium, the Maxwell equations simplify to:

$$
\begin{gathered}
\nabla \times \mathbf{e}=-j \omega \mu^{e} \mathbf{h}-\mathbf{m}, \\
\nabla \times \mathbf{h}=j \omega \varepsilon^{e} \mathbf{e}+\mathbf{j}, \\
\nabla \cdot \mathbf{e}=\frac{\rho_{e}}{\varepsilon^{e}}
\end{gathered}
$$

and

$$
\nabla \cdot \mathbf{h}=\frac{\rho_{m}}{\mu^{e}} .
$$

Here, $\mathbf{e}$ and $\mathbf{h}$ are the total electric and magnetic field, respectively. The electric and magnetic charges are given by $\rho_{e}$ and $\rho_{m}$. The externally applied currents on $\Gamma$ satisfy:

$$
\mathbf{j}=\mathbf{u}_{n} \times \mathbf{h}=\mathbf{u}_{n} \times\left(\mathbf{h}^{i}+\mathbf{h}^{s}\right)
$$


and

$$
\mathbf{m}=-\mathbf{u}_{n} \times \mathbf{e}=-\mathbf{u}_{n} \times\left(\mathbf{e}^{i}+\mathbf{e}^{s}\right),
$$

with $\mathbf{u}_{n}$ the normal on $\Gamma$ pointing towards $\Omega^{e}$. The surface equivalence principle states that this choice of currents generates the correct scattered fields in $\Omega^{e}$ and null fields in $\Omega^{i}$.

From (1)-(4) one can derive an expression for $\mathbf{e}$ and $\mathbf{h}$ as a function of the magnetic vector potential $\mathbf{A}$ and the electric vector potential $\mathbf{F}$ [18]. First, assume $\rho_{m}=\mathbf{m}=0$. In this case, the electromagnetic fields $\left(\mathbf{e}_{A}, \mathbf{h}_{A}\right)$, generated by $\rho_{e}$ and $\mathbf{j}$ are found as a function of the magnetic potential $\mathbf{A}$. They can be written as follows:

$$
\begin{gathered}
\mathbf{e}_{\mathbf{A}}=-j \omega \mathbf{A}+\frac{1}{j \omega \varepsilon^{e} \mu^{e}} \nabla(\nabla \cdot \mathbf{A}), \\
\mathbf{h}_{\mathbf{A}}=\frac{1}{\mu^{e}} \nabla \times \mathbf{A}
\end{gathered}
$$

where $A$ is the solution of

$$
\nabla^{2} \mathbf{A}+\left(k^{e}\right)^{2} \mathbf{A}=-\mu^{e} \mathbf{j}
$$

after applying the Lorentz gauge. In (9), $k^{e}=\omega \sqrt{\varepsilon^{e} \mu^{e}}$ is the wavenumber of the exterior region. Similarly, by assuming that $\rho_{e}=\mathbf{j}=0$, one can determine the electromagnetic fields $\left(\mathbf{e}_{F}, \mathbf{h}_{F}\right)$, generated by $\rho_{m}$ and $\mathbf{m}$. They can be written as a function of the electric potential $\mathbf{F}$, as follows:

$$
\begin{gathered}
\mathbf{h}_{\mathbf{F}}=-j \omega \mathbf{F}+\frac{1}{j \omega \varepsilon^{e} \mu^{e}} \nabla(\nabla \cdot \mathbf{F}), \\
\mathbf{e}_{\mathbf{F}}=\frac{-1}{\varepsilon^{e}} \nabla \times \mathbf{F},
\end{gathered}
$$

where $F$ is the solution of

$$
\nabla^{2} \mathbf{F}+\left(k^{e}\right)^{2} \mathbf{F}=-\varepsilon^{e} \mathbf{m}
$$

The solutions of the Helmholtz equations in (9) and (12) are obtained by introducing the Green function. After superposition of $\left(\mathbf{e}_{A}, \mathbf{h}_{A}\right)$ and $\left(\mathbf{e}_{F}, \mathbf{h}_{F}\right)$, one can derive the tangential scattered electromagnetic fields on the boundary $\Gamma$ generated by $\mathbf{j}$ and $\mathbf{m}$ : 


$$
\lim _{\mathbf{r} \in \Omega^{e} \rightarrow \Gamma}\left(\begin{array}{c}
-\mathbf{u}_{n} \times \mathbf{e}^{s} \\
\mathbf{u}_{n} \times \mathbf{h}^{s}
\end{array}\right)=\left(\begin{array}{cc}
\mathcal{K}_{k^{e}}+\frac{\mathcal{I}}{2} & -\eta^{e} \mathcal{T}_{k} \\
\frac{1}{\eta^{e}} \mathcal{T}_{k^{e}} & \mathcal{K}_{k^{e}}+\frac{\mathcal{I}}{2}
\end{array}\right)\left(\begin{array}{c}
\mathbf{m} \\
\mathbf{j}
\end{array}\right),
$$

with the impedance $\eta^{e, i}=\sqrt{\mu^{e, i} / \varepsilon^{e, i}}$, identity operator $\mathcal{I}$, and the integral operators $\mathcal{T}_{k^{e, i}}$ and $\mathcal{K}_{k^{e, i}}$ defined as

$$
\begin{aligned}
\mathcal{T}_{k^{e, i}}(\boldsymbol{x})(\mathbf{r})= & -j k^{e, i} \mathbf{u}_{n} \times \int_{\Gamma} \frac{e^{-j k^{e, i}\left|\boldsymbol{r}-\boldsymbol{r}^{\prime}\right|}}{4 \pi\left|\boldsymbol{r}-\boldsymbol{r}^{\prime}\right|} \boldsymbol{x}\left(\boldsymbol{r}^{\prime}\right) \mathrm{d} \boldsymbol{r}^{\prime} \\
& +\frac{1}{j k^{e, i}} \mathbf{u}_{n} \times p \cdot v \cdot \int_{\Gamma} \nabla \frac{e^{-j k^{e, i}\left|\boldsymbol{r}-\boldsymbol{r}^{\prime}\right|}}{4 \pi\left|\boldsymbol{r}-\boldsymbol{r}^{\prime}\right|} \nabla^{\prime} \cdot \boldsymbol{x}\left(\boldsymbol{r}^{\prime}\right) \mathrm{d} \boldsymbol{r}^{\prime}
\end{aligned}
$$

and

$$
\mathcal{K}_{k^{e, i}}(\boldsymbol{x})(\mathbf{r})=\mathbf{u}_{n} \times p . v . \int_{\Gamma} \nabla \times \frac{e^{-j k^{e, i}\left|\boldsymbol{r}-\boldsymbol{r}^{\prime}\right|}}{4 \pi\left|\boldsymbol{r}-\boldsymbol{r}^{\prime}\right|} \boldsymbol{x}\left(\boldsymbol{r}^{\prime}\right) \mathrm{d} \boldsymbol{r}^{\prime},
$$

with $k^{i}=\omega \sqrt{\varepsilon^{i} \mu^{i}}$, the wavenumber of the interior region. The notation $P(x)(r)$ in (14) and (15) denotes that an operator $P$ acts on function $x$, which depends on the variable $\mathbf{r}$. The principal value is designated by p.v.

Furthermore, the surface equivalence principle states that the fields in $\Omega^{i}$ are generated by $-\mathbf{j}$ and $-\mathbf{m}$, consequently resulting in zero fields in $\Omega^{e}$. This leads to the following relation:

$$
\lim _{\mathbf{r} \in \Omega^{i} \rightarrow \Gamma}\left(\begin{array}{c}
-\mathbf{u}_{n} \times \mathbf{e}^{s} \\
\mathbf{u}_{n} \times \mathbf{h}^{s}
\end{array}\right)=\left(\begin{array}{cc}
-\mathcal{K}_{k^{i}}+\frac{\mathcal{I}}{2} & \eta^{i} \mathcal{T}_{k^{i}} \\
-\frac{1}{\eta^{i}} \mathcal{T}_{k^{i}} & -\mathcal{K}_{k^{i}}+\frac{\mathcal{I}}{2}
\end{array}\right)\left(\begin{array}{c}
\mathbf{m} \\
\mathbf{j}
\end{array}\right) .
$$

The scattered tangential fields in the left hand side of (16) are equal to the total tangential fields, since we assume that there are no sources inside $\Omega^{i}$.

\subsection{CP-PMCHWT Equation}

In this section, the derivation of the CP-PMCHWT equation is summarized. This method will be employed in Section 5 as numerical validation of the results of the novel method. The PMCHWT formulation is obtained by subtracting (16) from (13). We obtain $[2,3]$ :

$$
P\left(\begin{array}{c}
\mathbf{m} \\
\mathbf{j}
\end{array}\right)=\left(\begin{array}{cc}
\mathcal{K}_{k^{e}}+\mathcal{K}_{k^{i}} & -\eta^{e} \mathcal{T}_{k^{e}}-\eta^{i} \mathcal{T}_{k^{i}} \\
\frac{\mathcal{T}_{k} e}{\eta^{e}}+\frac{\mathcal{T}_{k^{i}}}{\eta^{i}} & \mathcal{K}_{k^{e}}-\mathcal{K}_{k^{i}}
\end{array}\right)\left(\begin{array}{c}
\mathbf{m} \\
\mathbf{j}
\end{array}\right)=\left(\begin{array}{c}
\mathbf{u}_{n} \times \mathbf{e}^{i} \\
-\mathbf{u}_{n} \times \mathbf{h}^{i}
\end{array}\right)
$$

This equation is uniquely solvable. However, the eigenvalue spectrum of the system matrix, $\mathrm{P}$, in (17) is not bounded from below nor above, meaning that 
dense-mesh breakdown will occur. This leads to a slow iterative convergence and inaccurate solutions when solving the discretized problem. We may alleviate this problem by introducing a CP for the PMCHWT equation:

$$
P^{2}\left(\begin{array}{c}
\mathbf{m} \\
\mathbf{j}
\end{array}\right)=P\left(\begin{array}{c}
\mathbf{u}_{n} \times \mathbf{e}^{i} \\
-\mathbf{u}_{n} \times \mathbf{h}^{i}
\end{array}\right) .
$$

It can be shown [3] that the eigenvalues of the CP-PMCHWT accumulate at $-k^{i} /\left(4 k^{e}\right)$ and $-k^{e} /\left(4 k^{i}\right)$. The introduction of this preconditioner hence resolves the issues for low dielectric contrast media, as the system matrix will be well-posed and bounded. For high contrast media, $\left|k^{i}\right|$ will become large, and the accumulation points will tend to infinity and zero, respectively. This is detrimental for the conditioning of the CP-PMCHWT. Hence, a novel formulation is needed, with an operator that is well-posed and continuous in the limit for $\left|k^{i}\right|$ going to infinity. The derivation of a novel formulation that remains well-conditioned for high magnetic contrast media is given in Section 2.2. The derivation of a comparable method for high dielectric contrast media [17] is described in Section 2.3.

\subsection{Derivation of a CP for high magnetic contrasts}

In this section, a novel formulation that remains well-conditioned for high magnetic contrasts is derived. From (16), taking into account (5) and (6), we obtain the following relation for the tangential fields on $\Gamma$, if we approach this boundary from the inside:

$$
\lim _{\mathbf{r} \in \Omega^{i} \rightarrow \Gamma}\left(\mathbf{u}_{n} \times \mathbf{h}\right)=-\frac{\mathcal{T}_{k^{i}}}{\eta^{i}}\left(-\mathbf{u}_{n} \times \mathbf{e}\right)+\left(-\mathcal{K}_{k^{i}}+\frac{\mathcal{I}}{2}\right)\left(\mathbf{u}_{n} \times \mathbf{h}\right) .
$$

This allows us to derive an exact NtD operator $\mathcal{Y}_{k^{i}}$, with the property

$$
\lim _{\mathbf{r} \in \Omega^{i} \rightarrow \Gamma} \mathcal{Y}_{k^{i}}\left(\mathbf{u}_{n} \times \mathbf{h}\right)=-\mathbf{u}_{n} \times \mathbf{e}
$$

which is defined as

$$
\mathcal{Y}_{k^{i}}=-\eta^{i} \mathcal{T}_{k^{i}}^{-1}\left(\mathcal{K}_{k^{i}}+\frac{\mathcal{I}}{2}\right)
$$

It is assumed that $k^{i}$ is not a resonant wavenumber of the scattering object, such that $\mathcal{Y}_{k^{i}}$ exists and is unique [19].

At this point, we introduce a virtual electric current $\mathbf{J}$ on $\Gamma$, replacing $\mathbf{j}$ and $\mathbf{m}$ (see Fig. 2), and we impose that this source generates the same scattered electromagnetic fields in $\Omega^{e}$ as in the original scattering problem 


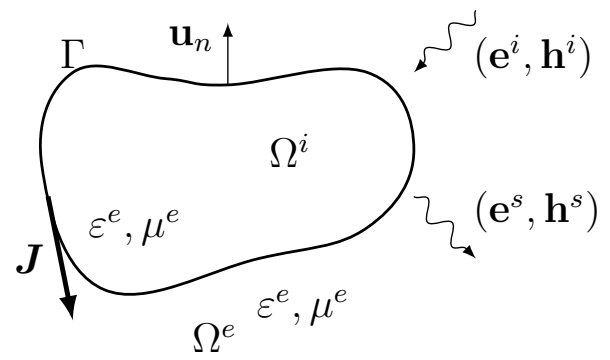

Figure 2: The object is filled with the medium of $\Omega_{e}$ and a surface current density $\mathbf{J}$, replacing $\mathbf{j}$ and $\mathbf{m}$, and generating the same scattered fields $\left(\boldsymbol{e}^{s}, \boldsymbol{h}^{s}\right)$ in $\Omega^{e}$ of Fig. 1(a), is introduced on $\Gamma$.

in Fig. 1(a). This means that the scattered electromagnetic fields in $\Omega^{e}$ are derived from the magnetic vector potential $\mathbf{A}$ in (7)-(9), with $\mathbf{F}=\mathbf{0}$. By using (13) and (20), and by taking into account the incident field in $\Omega^{e}$ and the continuity of the tangential fields across $\Gamma$, following relation is found:

$$
\lim _{\mathbf{r} \in \Omega^{e} \rightarrow \Gamma}\left(\begin{array}{c}
\mathcal{Y}_{k^{i}}\left(\mathbf{u}_{n} \times \mathbf{h}\right) \\
\mathbf{u}_{n} \times \mathbf{h}
\end{array}\right)=\left(\begin{array}{cc}
\mathcal{K}_{k^{e}}+\frac{\mathcal{I}}{2} & -\eta^{e} \mathcal{T}_{k^{e}} \\
\frac{1}{\eta^{e}} \mathcal{T}_{k^{e}} & \mathcal{K}_{k^{e}}+\frac{\mathcal{I}}{2}
\end{array}\right)\left(\begin{array}{l}
\mathbf{0} \\
\mathbf{J}
\end{array}\right)+\left(\begin{array}{c}
-\mathbf{u}_{n} \times \mathbf{e}^{i} \\
\mathbf{u}_{n} \times \mathbf{h}^{i}
\end{array}\right),
$$

with $\mathbf{0}$ the magnetic current, which is put to zero.

Notice that, in general, $\mathbf{J}$ is no longer equal to $\mathbf{j}=\mathbf{u}_{n} \times \mathbf{h}$. Hence, $\mathbf{u}_{n} \times \mathbf{h}$, which no longer generates scattered fields, is also an unknown in (22). The scattered fields are, hence, solely generated by $\mathbf{J}$. We can now rewrite (22) as follows:

$$
\lim _{\mathbf{r} \in \Omega^{e} \rightarrow \Gamma}\left(\begin{array}{cc}
\mathcal{K}_{k^{e}}+\frac{\mathcal{I}}{2} & -\mathcal{I} \\
-\eta^{e} \mathcal{T}_{k^{e}} & -\mathcal{Y}_{k^{i}}
\end{array}\right)\left(\begin{array}{c}
\mathbf{J} \\
\mathbf{u}_{n} \times \mathbf{h}
\end{array}\right)=\left(\begin{array}{c}
-\mathbf{u}_{n} \times \mathbf{h}^{i} \\
\mathbf{u}_{n} \times \mathbf{e}^{i}
\end{array}\right) .
$$

The equations in (23) should not be solved for $\mathbf{J}$ in this form, as the resulting system matrix after discretization would be ill-conditioned and would lead to a slow convergence of the Krylov iterative solution. Furthermore, (21) would have to be determined explicitly, which has to be avoided due to the presence of the inverse $\mathcal{T}_{k^{i}}^{-1}$ operator, leading to a computational complexity of $O\left(N^{3}\right)$.

These problems can be remedied by introducing following Calderón preconditioner:

$$
\mathcal{C}=\left(\begin{array}{cc}
\mathcal{I} & 0 \\
0 & \frac{1}{\eta^{i}} \mathcal{T}_{k^{i}}
\end{array}\right)
$$


After left multiplication of (23) with (24), and by using (21), we get:

$$
\lim _{\mathbf{r} \in \Omega^{e} \rightarrow \Gamma}\left(\begin{array}{cc}
\mathcal{K}_{k^{e}}+\frac{\mathcal{I}}{2} & -\mathcal{I} \\
-\frac{\eta^{e}}{\eta^{i}} \mathcal{T}_{k^{i}} \mathcal{T}_{k^{e}} & \mathcal{K}_{k^{i}}+\frac{\mathcal{I}}{2}
\end{array}\right)\left(\begin{array}{c}
\mathbf{J} \\
\mathbf{u}_{n} \times \mathbf{h}
\end{array}\right)=\left(\begin{array}{c}
-\mathbf{u}_{n} \times \mathbf{h}^{i} \\
\frac{1}{\eta^{i}} \mathcal{T}_{k^{i}}\left(\mathbf{u}_{n} \times \mathbf{e}^{i}\right)
\end{array}\right)
$$

In Section 4, it will be shown that the single-source equations in (25) lead to a well-conditioned system matrix after discretization for high magnetic contrasts. Furthermore, $(25)$ can be solved with a complexity of $O\left(N_{\text {iter }} N \log N\right)$ after implementation of the multilevel fast multipole method (MLFMM) [20].

The downside of this novel formulation is that it does not alleviate the ill-conditioning for problems involving high dielectric contrasts. However, in Section 2.3, a formulation that remains well-conditioned for high dielectric contrasts is summarized. The full derivation is given in [17].

\subsection{CP for high dielectric contrasts}

From (16), taking into account (5) and (6), we first obtain an expression for the inverse of the NtD operator - the DtN operator - introduced in (21):

$$
\mathcal{Y}_{k^{i}}^{-1}=\frac{1}{\eta^{i}} \mathcal{T}_{k^{i}}^{-1}\left(\mathcal{K}_{k^{i}}+\frac{\mathcal{I}}{2}\right)
$$

which has the property

$$
\lim _{\mathbf{r} \in \Omega^{i} \rightarrow \Gamma} \mathcal{Y}_{k^{i}}^{-1}\left(-\mathbf{u}_{n} \times \mathbf{e}\right)=\mathbf{u}_{n} \times \mathbf{h} .
$$

We next assume that the scattered electromagnetic fields in $\Omega^{e}$ are generated by a virtual magnetic current $\mathbf{M}$ on $\Gamma$. The virtual electric current $\mathbf{J}$

is set to zero. After some calculations [17], the following system of equations is found:

$$
\lim _{\mathbf{r} \in \Omega^{e} \rightarrow \Gamma}\left(\begin{array}{cc}
\mathcal{K}_{k^{e}}+\frac{\mathcal{I}}{2} & -\mathcal{I} \\
\frac{1}{\eta^{e}} \mathcal{T}_{k^{e}} & -\mathcal{Y}_{k^{i}}^{-1}
\end{array}\right)\left(\begin{array}{c}
\mathbf{M} \\
-\mathbf{u}_{n} \times \mathbf{e}
\end{array}\right)=\left(\begin{array}{c}
\mathbf{u}_{n} \times \mathbf{e}^{i} \\
-\mathbf{u}_{n} \times \mathbf{h}^{i}
\end{array}\right) .
$$

After preconditioning (28) with the CP

$$
\mathcal{C}=\left(\begin{array}{cc}
\mathcal{I} & 0 \\
0 & -\eta^{i} \mathcal{T}_{k^{i}}
\end{array}\right)
$$

we finally obtain the following matrix system:

$$
\lim _{\mathbf{r} \in \Omega^{e} \rightarrow \Gamma}\left(\begin{array}{cc}
\mathcal{K}_{k^{e}}+\frac{\mathcal{I}}{2} & -\mathcal{I} \\
-\frac{\eta^{i}}{\eta^{e}} \mathcal{T}_{k^{i}} \mathcal{T}_{k^{e}} & \mathcal{K}_{k^{i}}+\frac{\mathcal{I}}{2}
\end{array}\right)\left(\begin{array}{c}
\mathbf{M} \\
-\mathbf{u}_{n} \times \mathbf{e}
\end{array}\right)=\left(\begin{array}{c}
\mathbf{u}_{n} \times \mathbf{e}^{i} \\
\eta^{i} \mathcal{T}_{k^{i}}\left(\mathbf{u}_{n} \times \mathbf{h}^{i}\right)
\end{array}\right)
$$


It can be proven [17] that this method remains well-conditioned when scattering objects with high dielectric contrasts and high conductivities are involved. However, for high magnetic contrasts, the method becomes ill-conditioned. For such problems, the formulation in Section 2.2 can be employed.

\section{Discretization}

In this section, the discretization of the integral equations of Section 2.2 is described. The current $\mathbf{J}$ and tangential magnetic field $\mathbf{u}_{n} \times \mathbf{h}$, both belonging to the Sobolev space $H_{d i v}^{-\frac{1}{2}}(\Gamma)[21]$, have to be expanded in a set of basis functions $\left\{\mathbf{a}_{i}\right\}_{1 \leq i \leq N}$, belonging to the same space. The RWG basis functions are chosen, hence:

$$
\begin{gathered}
\mathbf{J} \approx \sum_{i=1}^{N}(\boldsymbol{\alpha})_{i} \mathbf{a}_{i} \\
\lim _{\mathbf{r} \in \Omega^{e} \rightarrow \Gamma}\left(\mathbf{u}_{n} \times \mathbf{h}\right) \approx \sum_{i=1}^{N}(\boldsymbol{\beta})_{i} \mathbf{a}_{i},
\end{gathered}
$$

with $\boldsymbol{\alpha}$ and $\boldsymbol{\beta}$ collecting the expansion coefficients of $\mathbf{J}$ and $\mathbf{u}_{n} \times \mathbf{h}$, respectively. Testing the identity operator, and consequently also $\mathcal{K}_{k^{i}}$ and $\mathcal{K}_{k^{e}}$, with rotated BC basis functions $\left(\left\{\mathbf{u}_{n} \times \mathbf{b}_{i}\right\}\right)_{1 \leq i \leq N} \in H_{\text {curl }}^{-\frac{1}{2}}(\Gamma)$ leads to more accurate results, also for nonsmooth boundaries, when compared to the original method, which involves expansion and testing of these terms with RWG functions $[22,23,24]$. To test $\mathcal{T}_{k^{e}}$, rotated RWG functions $\left(\left\{\mathbf{u}_{n} \times \mathbf{a}_{i}\right\}\right)_{1 \leq i \leq N} \in H_{\text {curl }}^{-\frac{1}{2}}(\Gamma)$ should be used. $\mathcal{T}_{k^{i}}$ is expanded into BC basis functions $\left(\left\{\mathbf{b}_{i}\right\}\right)_{1 \leq i \leq N} \in H_{d i v}^{-\frac{1}{2}}(\Gamma)$. This operator should be tested with rotated $\mathrm{BC}$ functions. The discretized equations can now be written as

$$
\left(\begin{array}{cc}
K_{k^{e}}+\frac{1}{2} G & -G \\
-\frac{\eta^{e}}{\eta^{i}} T_{k^{i}}\left(-G^{-1}\right)^{T} T_{k^{e}} & K_{k^{i}}+\frac{1}{2} G
\end{array}\right)\left(\begin{array}{l}
\boldsymbol{\alpha} \\
\boldsymbol{\beta}
\end{array}\right)=\left(\begin{array}{c}
-\mathbf{H}^{i} \\
\frac{1}{\eta^{i}} T_{k^{i}}\left(-G^{-1}\right)^{T} \mathbf{E}^{i}
\end{array}\right),
$$

with

$$
\begin{aligned}
\left(T_{k^{e}}\right)_{i j} & =\int_{\Gamma}\left(\mathbf{u}_{n}(\boldsymbol{r}) \times \boldsymbol{a}_{i}(\boldsymbol{r})\right) \cdot \mathcal{T}_{k^{e}}\left(\boldsymbol{a}_{j}\right)(\boldsymbol{r}) \mathrm{d} \boldsymbol{r} \\
\left(T_{k^{i}}\right)_{i j} & =\int_{\Gamma}\left(\mathbf{u}_{n}(\boldsymbol{r}) \times \boldsymbol{b}_{i}(\boldsymbol{r})\right) \cdot \mathcal{T}_{k^{i}}\left(\boldsymbol{b}_{j}\right)(\boldsymbol{r}) \mathrm{d} \boldsymbol{r}
\end{aligned}
$$




$$
\begin{gathered}
\left(K_{k^{e}}\right)_{i j}=\int_{\Gamma}\left(\mathbf{u}_{n}(\boldsymbol{r}) \times \boldsymbol{b}_{i}(\boldsymbol{r})\right) \cdot \mathcal{K}_{k^{e}}\left(\boldsymbol{a}_{j}\right)(\boldsymbol{r}) \mathrm{d} \boldsymbol{r}, \\
\left(K_{k^{i}}\right)_{i j}=\int_{\Gamma}\left(\mathbf{u}_{n}(\boldsymbol{r}) \times \boldsymbol{b}_{i}(\boldsymbol{r})\right) \cdot \mathcal{K}_{k^{i}}\left(\boldsymbol{a}_{j}\right)(\boldsymbol{r}) \mathrm{d} \boldsymbol{r}, \\
(G)_{i j}=\int_{\Gamma}\left(\mathbf{u}_{n}(\boldsymbol{r}) \times \boldsymbol{b}_{i}(\boldsymbol{r})\right) \cdot \boldsymbol{a}_{j}(\boldsymbol{r}) \mathrm{d} \boldsymbol{r}, \\
\mathbf{E}_{i}^{i}=\int_{\Gamma}\left(\mathbf{u}_{n}(\boldsymbol{r}) \times \boldsymbol{a}_{i}(\boldsymbol{r})\right) \cdot\left(\mathbf{u}_{n}(\boldsymbol{r}) \times \boldsymbol{e}(\boldsymbol{r})\right) \mathrm{d} \boldsymbol{r}, \\
\mathbf{H}_{i}^{i}=\int_{\Gamma}\left(\mathbf{u}_{n}(\boldsymbol{r}) \times \boldsymbol{b}_{i}(\boldsymbol{r})\right) \cdot\left(\mathbf{u}_{n}(\boldsymbol{r}) \times \boldsymbol{h}(\boldsymbol{r})\right) \mathrm{d} \boldsymbol{r},
\end{gathered}
$$

for $1 \leq i, j \leq N$. The mixed Gram matrix $G$ arises after discretization of the identity operator $\mathcal{I}$, and links the basis functions to the test functions.

This derivation for one scattering object in a homogeneous medium can be extended to a formulation including multiple scatterers and junctions. The system matrix for $M$ objects will then have the following form: .

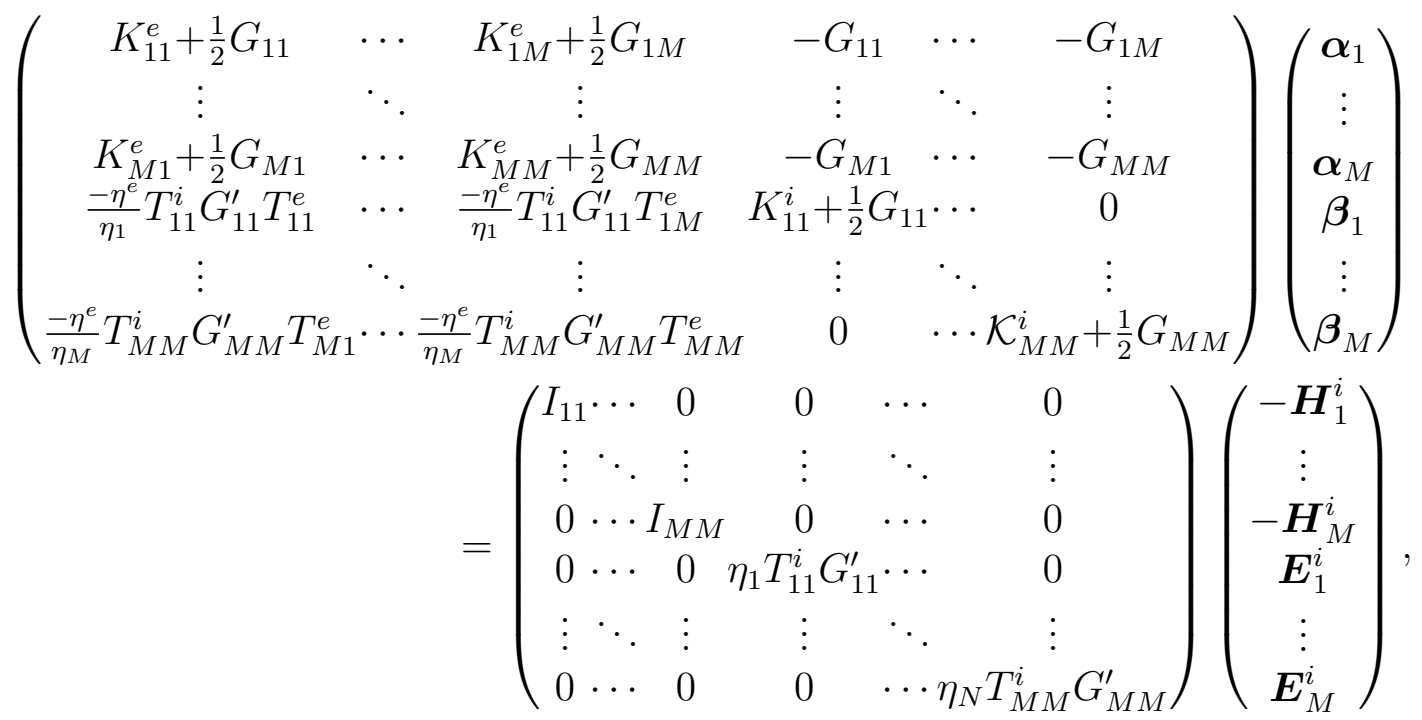

with $I_{a a}$ the unit matrix with dimensions equal the number of expansion functions of medium $a, \eta_{a}$ the impedance of medium $a$, and $G^{\prime}=\left(-G^{-1}\right)^{T}$. The subscripts $a$ and $b$ accompanying the $K, T$ and $I$ operator denote the restrictions on the operator's image and domain, meaning that test and expansion functions reside on object $a$ and $b$, respectively. 


\section{Spectral Properties}

In this section, we elaborate on the properties of the iterative solution of system matrix

$$
A=\left(\begin{array}{cc}
K_{k^{e}}+\frac{1}{2} G & -G \\
-\frac{\eta^{e}}{\eta^{i}} T_{k^{i}}\left(-G^{-1}\right)^{T} T_{k^{e}} & K_{k^{i}}+\frac{1}{2} G
\end{array}\right) .
$$

The behavior of the convergence of the solution for scattering problems including high magnetic contrasts is of particular interest. For this purpose, the position of the eigenvalue accumulation points of $A$ is derived below. First, notice that $A$ can be rewritten as

$$
A=\left(\begin{array}{cc}
\frac{1}{2} I & -I \\
-\frac{\eta^{e}}{\eta^{i}} T_{k^{i}}\left(-G^{-1}\right)^{T} T_{k^{e}} G^{-1} & \frac{1}{2} I
\end{array}\right)\left(\begin{array}{cc}
G & 0 \\
0 & G
\end{array}\right)+\left(\begin{array}{cc}
K_{k^{e}} & 0 \\
0 & K_{k^{i}}
\end{array}\right)
$$

with $I$ the $N \times N$ unit matrix.

The second term in the right hand side (rhs) of (43) is compact on smooth surfaces, since the block diagonal matrices are compact on smooth surfaces, and the off-diagonal matrix blocks are zero. For nonsmooth surfaces, the near-field interactions of $\mathcal{K}_{k}$ are no longer weak, which compromises the compactness of the operator [22]. However, in [23], it was shown that the mixed discretization scheme of $\mathcal{K}_{k}$, which is used in this paper, delivers a much better accuracy for nonsmooth geometries than the classical testing scheme of $\mathcal{K}_{k}$ with RWG functions. It can, hence, be assumed that the eigenvalues of the rightmost matrix in the rhs of (43) cluster at zero and are bounded. This term will consequently not have a meaningful influence on the eigenvalue accumulation points of $A[3]$.

The second matrix in the first term of the rhs of (43) is composed of two diagonal blocks containing the Gram matrix (38). This factor is an artifact of the discretization of (25) in nonorthonormal bases. Nevertheless, since $G$

is diagonally dominant and well-conditioned, this matrix will merely lead to a small perturbation of the eigenvalues of the first matrix in the rhs of (43). Hence, the spectral properties of $A$ will depend on the spectrum of

$$
B=\left(\begin{array}{cc}
\frac{1}{2} I & -I \\
-\frac{\eta^{e}}{\eta^{i}} T_{k^{i}}\left(-G^{-1}\right)^{T} T_{k^{e}} G^{-1} & \frac{1}{2} I
\end{array}\right) .
$$

The eigenvalues and, hence, the eigenvalue accumulation points $\lambda_{i}$ of $B$, can be found by solving $\operatorname{det}\left(B-\lambda_{i} I\right)=0$, which can be rewritten as: 


$$
\operatorname{det}\left[\left(\frac{1}{2}-\lambda_{i}\right)^{2} I-\frac{\eta^{e}}{\eta^{i}} T_{k^{i}}\left(-G^{-1}\right)^{T} T_{k^{e}} G^{-1}\right]=0 .
$$

The eigenvalue accumulation points $\xi_{i}$ of $T_{k^{i}}\left(-G^{-1}\right)^{T} T_{k^{e}} G^{-1}$ are known, and given by $\xi_{1}=\frac{-k^{i}}{4 k^{e}}$ and $\xi_{2}=\frac{-k^{e}}{4 k^{i}}[3]$. These accumulations points are solutions of

$$
\operatorname{det}\left[T_{k^{i}}\left(-G^{-1}\right)^{T} T_{k^{e}} G^{-1}-\xi_{i} I\right]=0,
$$

which can be rewritten as

$$
\left(\frac{\eta^{i}}{\eta^{e}}\right)^{N} \operatorname{det}\left[\left(\frac{\eta^{e}}{\eta^{i}} T_{k^{i}}\left(-G^{-1}\right)^{T} T_{k^{e}} G^{-1}\right)-\frac{\eta^{e}}{\eta^{i}} \xi_{i} I\right]=0 .
$$

After combining (45) and (47), and after filling in the values of $\xi_{i}$, we obtain:

$$
\left(\frac{1}{2}-\lambda_{i}\right)^{2}=-\frac{\varepsilon^{i}}{4 \varepsilon^{e}}
$$

and

$$
\left(\frac{1}{2}-\lambda_{i}\right)^{2}=-\frac{\mu^{e}}{4 \mu^{i}}
$$

of which the solutions are given by

$$
\lambda_{1, \pm}=\frac{1}{2} \pm \frac{1}{2} \sqrt{\frac{\varepsilon^{i}}{\varepsilon^{e}} j}
$$

and

$$
\lambda_{2, \pm}=\frac{1}{2} \pm \frac{1}{2} \sqrt{\frac{\mu^{e}}{\mu^{i}}} j .
$$

From (50) and (51), one can conclude that the eigenvalue accumulation points will not tend to zero or infinity given the following conditions. First, the exterior region should have a low relative permeability (e.g., vacuum). Second, the dielectric contrast of the object should not become arbitrary large. Last, the permittivity of the object should not tend to $-\varepsilon^{e}$, while its permeability should not tend to $-\mu^{e}$ or 0 . In these circumstances, it can be seen that the eigenvalue accumulation points do not go to zero or infinity for arbitrary low or high (complex) permeability, see Fig. 3. The system matrix is hence well-posed and bounded. In Fig. 4, the ratio of the eigenvalue accumulation points of the CP-PMCHWT equation is given for comparison. It should be noticed that even for relatively low material contrasts (e.g., 


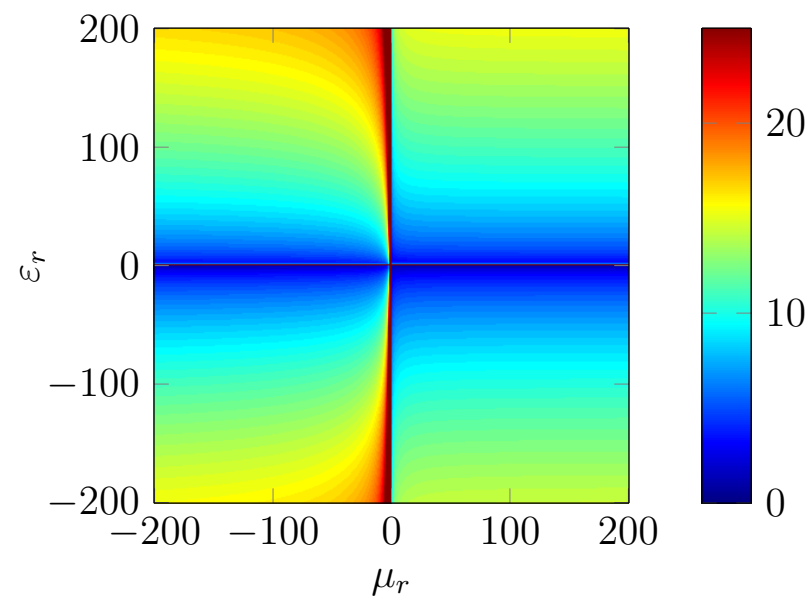

Figure 3: Ratio $\frac{\left|\lambda_{\max }\right|}{\left|\lambda_{\min }\right|}$ of the eigenvalue accumulation points of (44) as a function of the relative permittivity and permeability of an object embedded in free space. Similar results are obtained when $\mu_{r}$ and $\varepsilon_{r}$ are complex. This value remains approximately constant as a function of $\mu_{r}$, and rises proportionally to $\sqrt{\varepsilon_{r}}$, for $\mu_{r} \rightarrow \infty$. For $\mu_{r} \rightarrow-1, \mu_{r} \rightarrow 0$ and $\varepsilon_{r} \rightarrow-1$ and $\varepsilon_{r} \rightarrow \infty$, the system matrix becomes singular.

$\left.\varepsilon_{r}=5, \mu_{r}=5\right)$, the ratio already is an order of magnitude higher than that of (33).

In the absence of resonances, the corresponding convergence speed of the scattering problem will be fast. For high frequencies, resonances might occur. In this case, the eigenvalue accumulation points remain the same, but the radius around which the eigenvalues are scattered increases [3]. This will lead to a worse condition number and reduced accuracy in the immediate neighborhood of these resonance frequencies. The CP-PMCHWT method also suffers from physical resonances, but not from spurious ones, as it is a double-source formulation.

In [17], a similar method was developed that remained well conditioned for an arbitrary dielectric contrast. This dual method is valid under the condition that the external region has a low permittivity, and that the permeability of the object is low.

\section{Numerical Examples}

In this section, we present numerical examples to validate the effectiveness and accuracy of the proposed method. All systems are solved iteratively with 


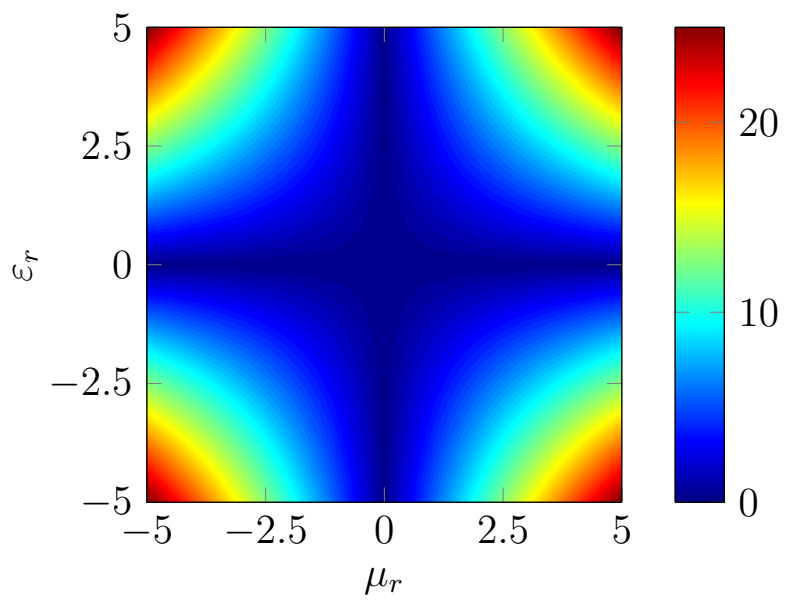

Figure 4: Ratio $\frac{\left|\lambda_{\max }\right|}{\left|\lambda_{\min }\right|}$ of the eigenvalue accumulation points of the CP-PMCHWT [3] method as a function of the relative permittivity and permeability of an object embedded in free space. $\frac{\left|\lambda_{\max }\right|}{\left|\lambda_{\min }\right|}$ increases quickly for increasing scatterer contrast, proportionally to $\varepsilon_{r} \mu_{r}$. Remark that the scale of both axes of this figure is orders of magnitude lower than that of Fig. 3. For $\varepsilon_{r}=\mu_{r}=200$ the ratio is already 3 orders of magnitude higher than that of the system matrix in (33). 


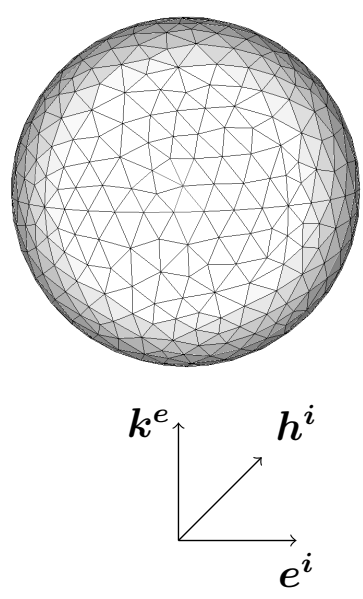

Figure 5: Electromagnetic scattering at a sphere with radius of $1 \mathrm{~m}$, at $1 \mathrm{MHz}$. A linearly polarized plane wave $\left(\boldsymbol{e}^{i}(\boldsymbol{r})=\boldsymbol{u}_{x} e^{-j k \boldsymbol{u}_{z} \cdot \boldsymbol{r}}\right)$ impinges on this sphere, which is consecutively filled with different materials. The far field RCS is evaluated in the xz-plane (spanned by $\boldsymbol{k}^{\boldsymbol{e}}$ and $\boldsymbol{e}^{\boldsymbol{i}}$ ), for $\theta$ varying from $0^{\circ}$ (towards bottom of sphere) to $180^{\circ}$ (towards top of sphere).

the generalized minimal residual (GMRES) method [25], without restarts, within a convergence threshold of $10^{-5}$. The CP-PMCHWT, which is used as comparison, is implemented as described in [26], Section II.D.

First, the accuracy, condition number and convergence speed of a simple scattering problem involving a homogeneous sphere in a homogeneous background medium is considered. The condition number is defined as the ratio of the highest and lowest singular value of the system matrix. A linearly polarized plane wave $\left(\boldsymbol{e}^{i}(\boldsymbol{r})=\boldsymbol{u}_{x} e^{-j k \boldsymbol{u}_{z} \cdot \boldsymbol{r}}\right)$ impinges on this sphere at a frequency of $1 \mathrm{MHz}$. We compare the numerical solution to the analytical Mie series solution [27]. In Fig. 6, the condition number of the system matrix corresponding to a spherical scatterer with a radius of $1 \mathrm{~m}$ is given for both (33) and the CP-PMCHWT method, as a function of magnetic contrast, for different mesh densities. These densities are defined by the mesh parameter $h=\lambda / \delta$, with $\lambda$ the wavelength of the impinging wave in vacuum, and $\delta$ the average mesh edge length. It can be seen that the condition number of the proposed method rises slightly as a function of scatterer permeability for low magnetic contrasts $\left(\mu_{r}<10\right)$. The condition number then converges to a stable, low value for high magnetic contrasts. It should be noticed that the 
condition number does not depend on the mesh density; dense-mesh breakdown does not occur. In comparison, the CP-PMCHWT method shows a stable condition number that is an order of magnitude higher than that of the proposed method for low magnetic contrasts $\left(\mu_{r}<10\right)$. Furthermore, for high magnetic contrasts, the condition number becomes proportional to the scatterer permeability. In Fig. 7, the corresponding number of iterations until convergence ( $\mathrm{rms}$ error $<10^{-5}$ ) are plotted for both methods as a function of scatterer permeability, for different mesh densities. We observe similar results as in Fig. 6, i.e., the convergence time increases slightly for low magnetic contrasts for the proposed method, and converges to a low value for high contrasts. The number of iterations until convergence of the CPPMCHWT method rises proportionally to the permeability of the scatterer, becoming higher than the proposed method when $\mu_{r} \approx 100$. For low material contrasts, the convergence speed of the CP-PMCHWT method is higher, even though it has a higher condition number than the proposed method, which might appear counterintuitive. In these circumstances, the condition number is not the decisive factor in the determination of the convergence speed. This is also influenced by the eigenvalue distribution of the system matrix, and not solely by the ratio of the largest and smallest eigenvalues. A similar behavior was observed in [17].

To test the accuracy of the proposed method, the radar cross sections (RCSs) of a sphere composed of a material with a high permeability, a metamaterial $\left(\varepsilon_{r}<0\right.$ and $\left.\mu_{r}<0\right)$ and a material with a high complex permeability are compared to the analytical Mie series solution in Fig. 8. There is a very good correspondence between analytical and numerical results, with a root mean square (rms) difference smaller than $0.1 \%$, calculated with the Lebedev quadrature [28], for the three investigated examples .

Next, simultaneous scattering at four objects is considered. The problem is visualized in Fig. 9. The accuracy of this solution may be investigated by first filling the cube, cuboid and cylinder with a vacuum, and the sphere with a dielectric material $\left(\varepsilon_{r}=4, \mu_{r}=4\right)$. In this way, the solution can again be compared to the Mie series solution, as the currents on the nonspherical objects should generate a zero scattered electromagnetic field in $\Omega^{e}$. The rms error stays below $2.5 \%$ for both simulated frequencies. This is more than the error examined for the single spherical scatterer in Fig. 8, which is due to the presence of sharp wedges on the vacuum objects (see Section 4). Nevertheless, this error remains rather small, and sufficiently accurate results are still obtained. 


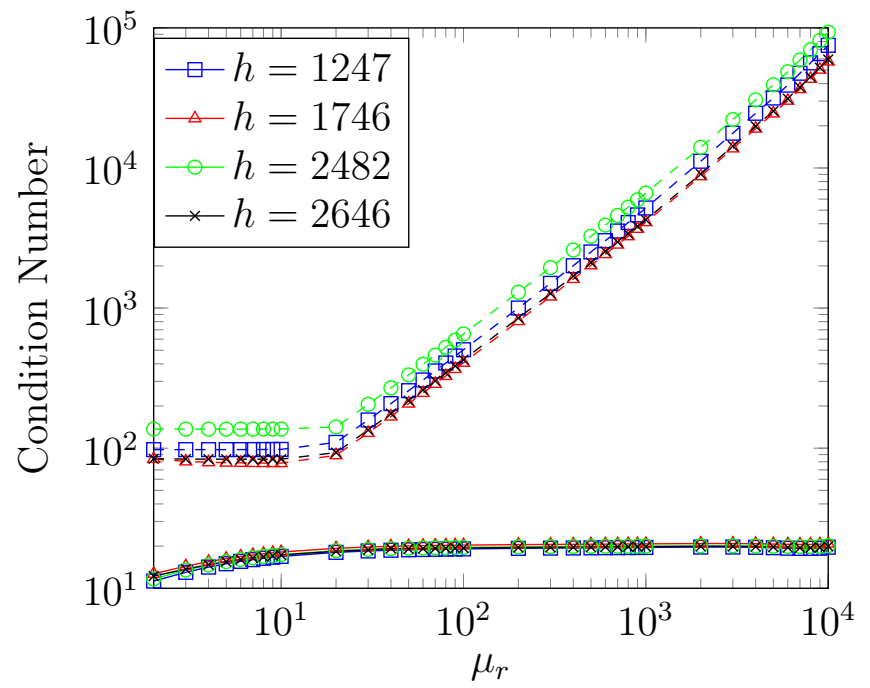

Figure 6: Condition number of the proposed method (full line) and the CP-PMCHWT method (dashed line) as a function of permeability (with $\varepsilon_{r}=1$ ), for a spherical scatterer with radius of $1 \mathrm{~m}$. The incoming plane wave $\boldsymbol{e}^{i}(\boldsymbol{r})=\boldsymbol{u}_{x} e^{-j k \boldsymbol{u}_{z} \cdot \boldsymbol{r}}$ impinges along the z-axis at a frequency of $1 \mathrm{MHz}$. Data are given for different mesh densities $h$.

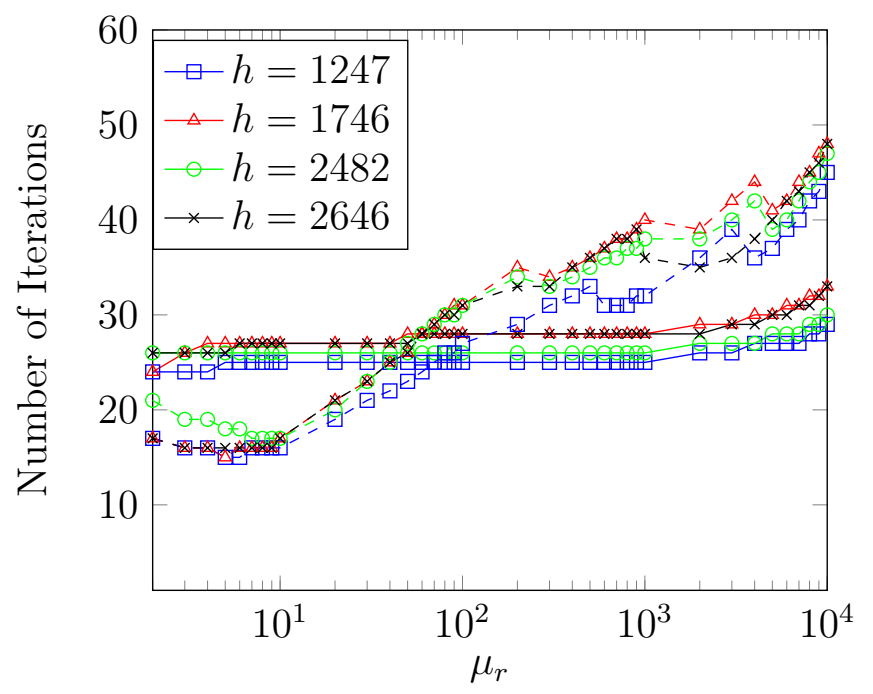

Figure 7: Number of iterations until convergence (residual error $<10^{-5}$ ) of the proposed method (full line) and the CP-PMCHWT method (dashed line) as a function of permeability (with $\varepsilon_{r}=1$ ) for a spherical scatterer with radius of $1 \mathrm{~m}$. The incoming plane wave $\boldsymbol{e}^{i}(\boldsymbol{r})=\boldsymbol{u}_{x} e^{-j k \boldsymbol{u}_{z} \cdot \boldsymbol{r}}$ impinges along the z-axis at a frequency of $1 \mathrm{MHz}$. Data are given for different mesh densities $h$. 


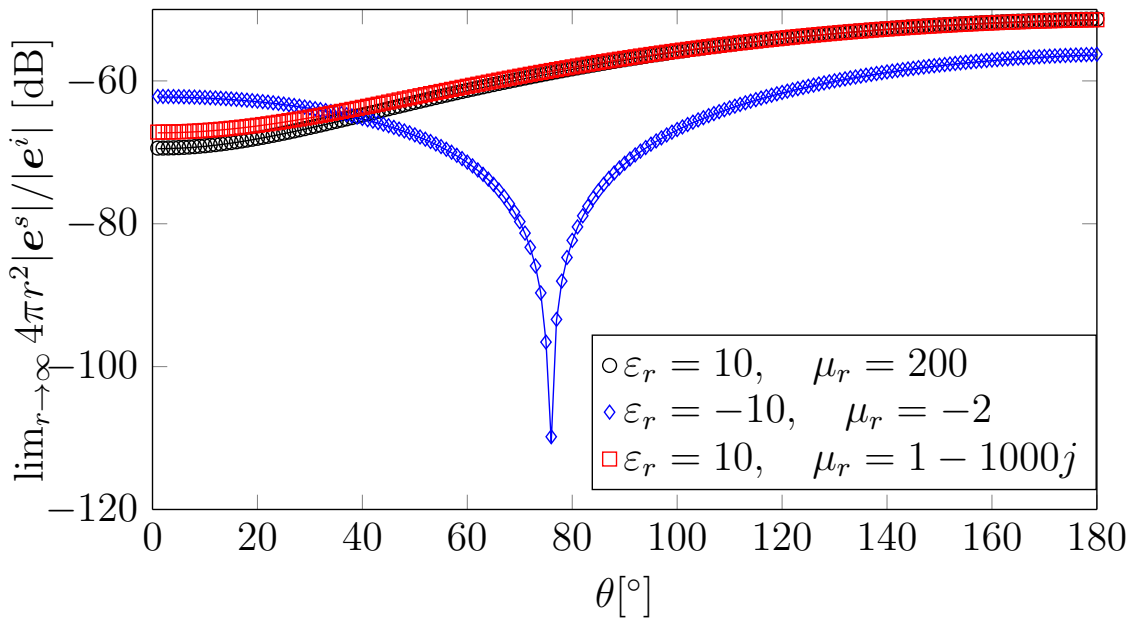

Figure 8: RCS of scattering at a sphere with radius of $1 \mathrm{~m}$ and mesh parameter $h=1920$, consecutively filled with three materials $\left(\varepsilon_{r}=10, \mu_{r}=200 / \varepsilon_{r}=-10, \mu_{r}=-2 /\right.$ $\left.\varepsilon_{r}=10, \mu_{r}=1-1000 j\right)$. The impinging plane wave is given by $\boldsymbol{e}^{i}(\boldsymbol{r})=\boldsymbol{u}_{x} e^{-j k \boldsymbol{u}_{z} \cdot \boldsymbol{r}}$, at a frequency of $1 \mathrm{MHz}$. The RCS is plotted in the xz-plane for $\theta$ varying over $180^{\circ}$. The results (symbols) are validated by the analytical Mie series expansion (line). The rms difference between both solutions is smaller than $0.1 \%$ for the three investigated cases.

Next, we fill all objects with a contrast material, as clarified in the caption of Fig. 9. In Fig. 10 and Fig. 11, the RCSs corresponding to this scattering problem are given for different frequencies, in the xz- and xy-plane, respectively. In Table 1, the condition number and number of iterations until convergence for this scattering problem are given, for different mesh densities. Notice that for all investigated cases, both the condition number and the convergence time remain low, and do not vary much when the mesh density increases.

\section{Conclusion}

In this paper, we have presented a novel, Calderón preconditioned singlesource BIE. It was proven that this formulation is well-conditioned for electromagnetic scattering problems including arbitrarily high magnetic contrasts. The conditioning, convergence speed and accuracy of this method were investigated for a wide range of scatterer permeabilities. For high magnetic contrasts, the system matrix of the proposed method is better conditioned and the iterative solution converges faster, when compared to the CP-PMCHWT 


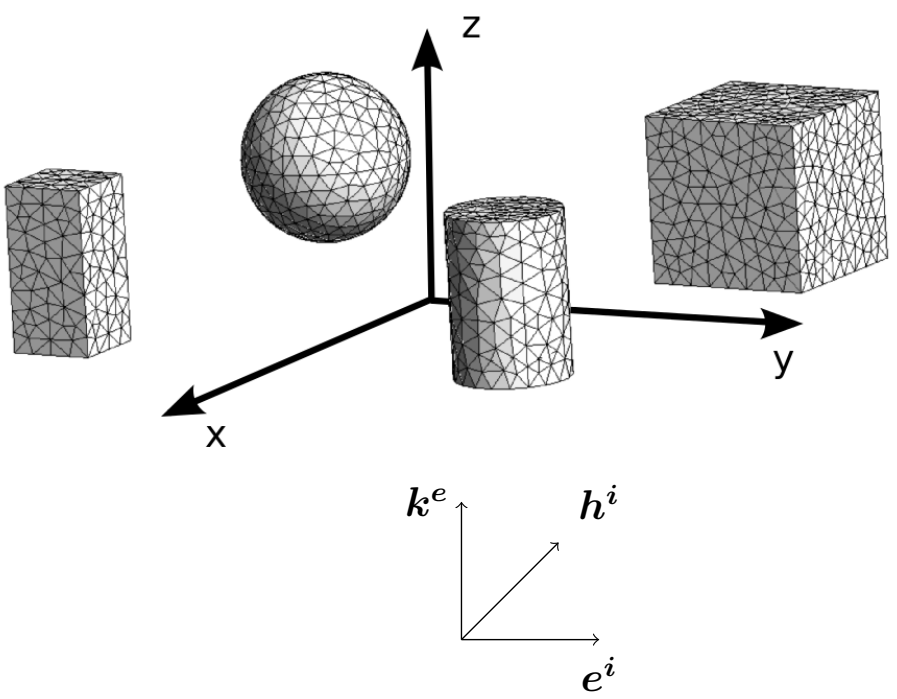

Figure 9: Electromagnetic scattering at four objects. A linearly polarized plane wave $\left(\boldsymbol{e}^{i}(\boldsymbol{r})=\boldsymbol{u}_{x} e^{-j k \boldsymbol{u}_{z} \cdot \boldsymbol{r}}\right)$ impinges on a sphere with a radius of $1 \mathrm{~m}\left(\varepsilon_{r}=4, \mu_{r}=4\right)$, a cube with a length of $2 \mathrm{~m}\left(\varepsilon_{r}=15, \mu_{r}=15\right)$, a cuboid with a height of $2 \mathrm{~m}$ and a length of $1 \mathrm{~m}\left(\varepsilon_{r}=4, \mu_{r}=100\right)$ and a cylinder with a radius of $0.5 \mathrm{~m}$ and a height of $2 \mathrm{~m}\left(\varepsilon_{r}=4\right.$, $\left.\mu_{r}=1-100 j\right)$.

\begin{tabular}{|c|c|c|c|}
\hline $\mathrm{h}$ & $\mathrm{f}$ & condition nr. & nr. of iter. \\
\hline \hline 75.49 & $10 \mathrm{MHz}$ & 266.63 & 213 \\
754.9 & $1 \mathrm{MHz}$ & 239.87 & 136 \\
\hline 83.39 & $10 \mathrm{MHz}$ & 287.39 & 194 \\
833.9 & $1 \mathrm{MHz}$ & 265.82 & 114 \\
\hline 100.6 & $10 \mathrm{MHz}$ & 305.01 & 212 \\
1006 & $1 \mathrm{MHz}$ & 258.22 & 128 \\
\hline 112.9 & $10 \mathrm{MHz}$ & 392.41 & 209 \\
1129 & $1 \mathrm{MHz}$ & 311.47 & 127 \\
\hline 132.3 & $10 \mathrm{MHz}$ & 287.87 & 205 \\
1323 & $1 \mathrm{MHz}$ & 283.98 & 121 \\
\hline
\end{tabular}

Table 1: Condition number and number of iterations until convergence (residual error $<10^{-5}$ ) for the scattering problem of Fig. 9, for different frequencies and mesh densities. 


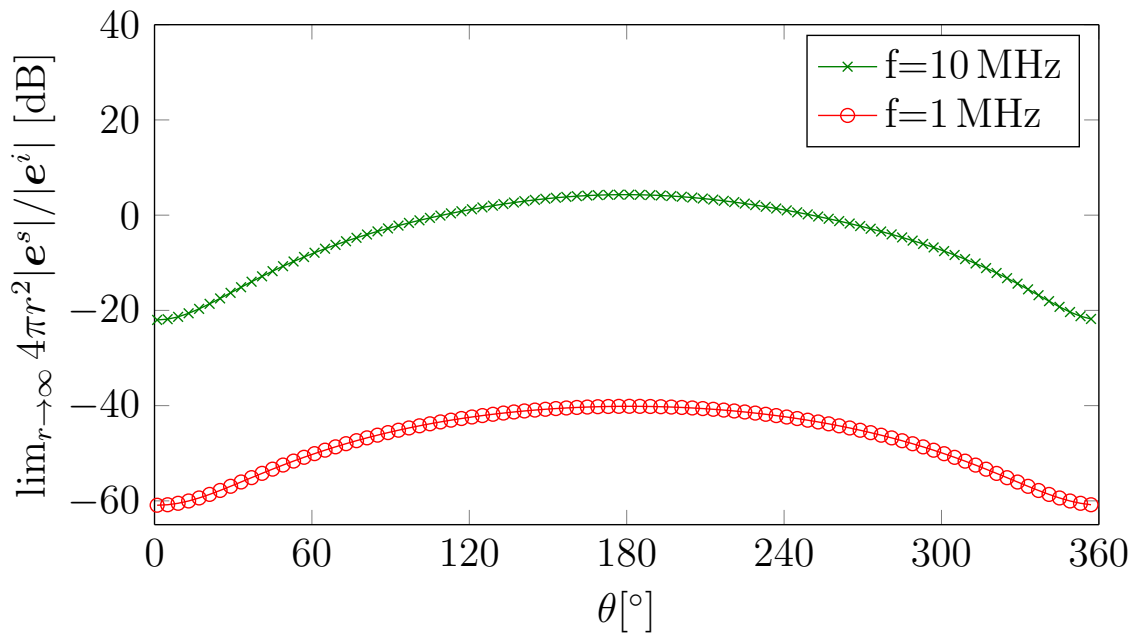

Figure 10: RCS of the scattering problem depicted in Fig. 9, for different frequencies of the incoming plane wave. The RCSs are plotted in the xz-plane, with $\theta=180^{\circ}$ in the direction of propagation of the impinging wave. The mesh parameter $h$ equals 1323 at $f=1 \mathrm{MHz}$ and 132.3 at $f=10 \mathrm{MHz}$, respectively.

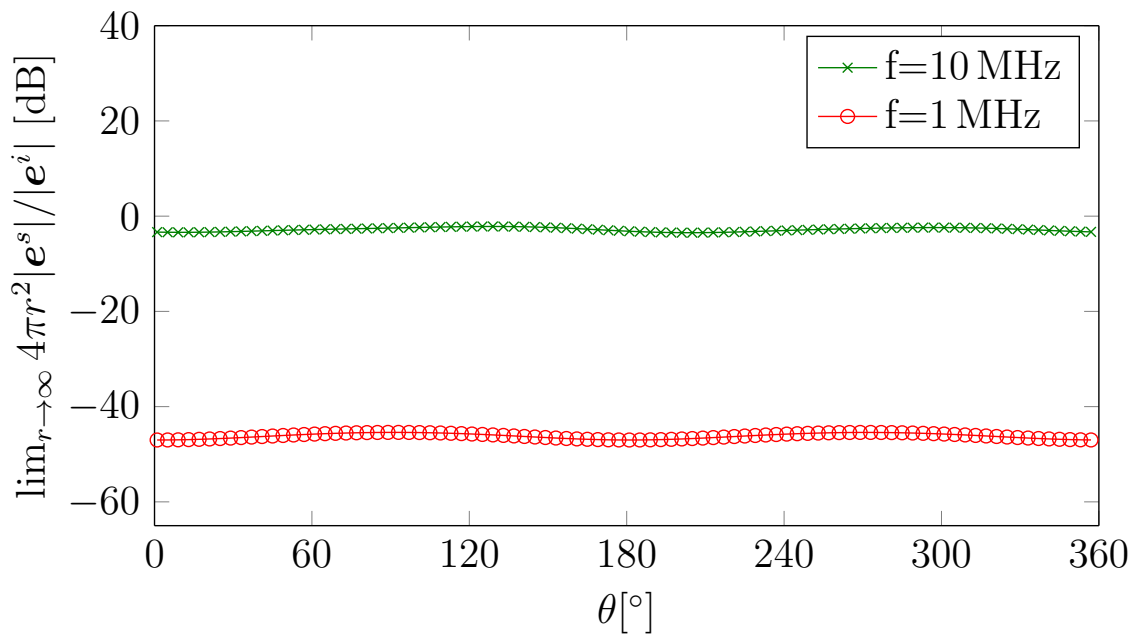

Figure 11: RCS of the scattering problem depicted in Fig. 9, for different frequencies of the incoming plane wave. The RCSs are plotted in the xy-plane, with $\theta=180^{\circ}$ in the direction of propagation of the impinging wave. The mesh parameter $h$ equals 1323 at $f=1 \mathrm{MHz}$ and 132.3 at $f=10 \mathrm{MHz}$, respectively. 
method.

\section{Acknowledgment}

The authors would like to thank the Research Foundation Flanders (FWO) for supporting this research.

[1] D. Dobbelaere, D. De Zutter, J. Van Hese, J. Sercu, T. Boonen, H. Rogier, A Calderón multiplicative preconditioner for the electromagnetic Poincaré-Steklov operator of a heterogeneous domain with scattering applications, Journal of Computational Physics 303 (2015) 355-371.

[2] A. Poggio, E. Miller, Chapter 4 - integral equation solutions of threedimensional scattering problems, in: Computer Techniques for Electromagnetics, International Series of Monographs in Electrical Engineering, Pergamon, 1973, pp. $159-264$.

[3] K. Cools, F. P. Andriulli, E. Michielssen, A Calderón multiplicative preconditioner for the PMCHWT integral equation, IEEE Transactions on Antennas and Propagation 59 (12) (2011) 4579-4587.

[4] O. Steinbach, W. Wendland, The construction of some efficient preconditioners in the boundary element method, Advances in Computational Mathematics 9 (1) (1998) 191-216.

[5] S. H. Christiansen, J.-C. Nédélec, A preconditioner for the electric field integral equation based on Calderón formulas, SIAM Journal on Numerical Analysis 40 (3) (2002) 1100-1135.

[6] F. P. Andriulli, K. Cools, H. Bağci, F. Olyslager, A. Buffa, S. Christiansen, E. Michielssen, A multiplicative Calderón preconditioner for the electric field integral equation, IEEE Transactions on Antennas and Propagation 56 (8) (2008) 2398-2412.

[7] J.-C. Nédélec, Acoustic and electromagnetic equations : integral representations for harmonic problems, Applied mathematical sciences, Springer, New York, 2001.

[8] A. Buffa, S. H. Christiansen, A dual finite element complex on the barycentric refinement, Mathematics of Computation 76 (260) (2007) 1743-1769. 
[9] S. Rao, D. Wilton, A. Glisson, Electromagnetic scattering by surfaces of arbitrary shape, IEEE Transactions on Antennas and Propagation 30 (3) (1982) 409-418.

[10] P. Ylä-Oijala, M. Taskinen, Well-conditioned Müller formulation for electromagnetic scattering by dielectric objects, IEEE Transactions on Antennas and Propagation 53 (10) (2005) 3316-3323.

[11] P. Ylä-Oijala, S. P. Kiminki, Challenges in developing efficient Calderón preconditioners for resonating or high material contrast penetrable objects, Journal of Computational and Applied Mathematics 289 (2015) 296 - 305, sixth International Conference on Advanced Computational Methods in Engineering (ACOMEN 2014).

[12] M. E. Bouajaji, X. Antoine, C. Geuzaine, Approximate local magneticto-electric surface operators for time-harmonic Maxwell's equations, Journal of Computational Physics 279 (2014) 241 - 260.

[13] M. E. Bouajaji, B. Thierry, X. Antoine, C. Geuzaine, A quasi-optimal domain decomposition algorithm for the time-harmonic Maxwell's equations, Journal of Computational Physics 294 (2015) 38 - 57.

[14] Y. Boubendir, X. Antoine, C. Geuzaine, A quasi-optimal nonoverlapping domain decomposition algorithm for the Helmholtz equation, Journal of Computational Physics 231 (2) (2012) 262 - 280.

[15] Y. Boubendir, V. Domínguez, D. Levadoux, C. Turc, Regularized combined field integral equations for acoustic transmission problems, SIAM Journal on Applied Mathematics 75 (3) (2015) 929-952.

[16] D. Levadoux, F. Millot, S. Pernet, A well-conditioned boundary integral equation for transmission problems of electromagnetism, Journal of Integral Equations and Applications 27 (3) (2015) 431-454.

[17] M. Gossye, M. Huynen, D. V. Ginste, D. D. Zutter, H. Rogier, A Calderón preconditioner for high dielectric contrast media, IEEE Transactions on Antennas and Propagation 66 (2) (2018) 808-818.

[18] C. Balanis, Advanced Engineering Electromagnetics, CourseSmart Series, Wiley, 2012. 
[19] L. Knockaert, D. D. Zutter, On the complex symmetry of the PoincaréSteklov operator, Progress In Electromagnetics Research B 7 (2008) 145-157.

[20] W. Chew, E. Michielssen, J. M. Song, J. M. Jin (Eds.), Fast and Efficient Algorithms in Computational Electromagnetics, Artech House, Inc., Norwood, MA, USA, 2001.

[21] R. A. Adams, J. J. F. Fournier, Sobolev spaces, Pure and applied mathematics, Elsevier, Amsterdam, Boston, Heildelberg, 2003.

[22] S. Yan, J. M. Jin, Z. Nie, Improving the accuracy of the second-kind Fredholm integral equations by using the Buffa-Christiansen functions, IEEE Transactions on Antennas and Propagation 59 (4) (2011) 12991310 .

[23] S. Yan, J. M. Jin, Z. Nie, Accuracy improvement of the second-kind integral equations for generally shaped objects, IEEE Transactions on Antennas and Propagation 61 (2) (2013) 788-797.

[24] K. Cools, F. P. Andriulli, D. D. Zutter, E. Michielssen, Accurate and conforming mixed discretization of the mfie, IEEE Antennas and Wireless Propagation Letters 10 (2011) 528-531.

[25] Y. Saad, M. H. Schultz, GMRES: A generalized minimal residual algorithm for solving nonsymmetric linear systems, SIAM Journal on Scientific and Statistical Computing 7 (3) (1986) 856-869.

[26] S. Yan, J. M. Jin, Z. Nie, A comparative study of Calderón preconditioners for PMCHWT equations, IEEE Transactions on Antennas and Propagation 58 (7) (2010) 2375-2383.

[27] J. A. Stratton, Electromagnetic Theory, IEEE Press series on Electromagnetic Wave Theory, Wiley-IEEE Press, 2007.

[28] V. Lebedev, Quadratures on a sphere, USSR Computational Mathematics and Mathematical Physics 16 (2) (1976) $10-24$. 